\title{
CHOICE OF FOOD SAMPLE IN EXAMINING THE MASTICATORY FUNCTION IN EDENTULOUS PATIENTS AND IN PATIENTS WITH REMOVABLE DENTURES
}

\author{
Desislava Konstantinova ${ }^{1}$, Mariana Dimova ${ }^{2}$ \\ ${ }^{1}$ Department of Prosthetic Dental Medicine and Orthodontics, Faculty of Dental Medicine, \\ Medical University of Varna, and ${ }^{2}$ Department of Prosthetic Dental Medicine, \\ Faculty of Dental Medicine, Medical University of Sofia
}

\begin{abstract}
A thorough dental prosthetic treatment must meet all three medico-biological criteria. Dental aesthetics becomes worthless if dentures do not meet the functional requirements. Therefore, the study of masticatory efficiency proves essential in the evaluation of dental prosthetic treatment. Based on recent literature review, objective methods for examining the masticatory function and choice of sample material were analysed.
\end{abstract}

Key words: food sample, test material, masticatory function, removable dental prostheses, literature survey

\section{INTRODUCTION}

Mastication is the process by which food is dispergated into smaller particles, thus increasing its surface area (8). As chewing continues, the food mixed with saliva reaches body temperature while turning it into a bolus. At the same time, pleasant sensations delivered by taste and smell satisfy basic human necessities.

Mastication comprises complex mechanical and physiological processes whereby all the parts of the chewing apparatus are involved. This makes it rather difficult to objectively study the masticatory function. Attempts for such a study have been carried out since the beginning of the last century onwards (23). Some researchers $(1,3,4,7,19,20)$ still continue to explore new methods in order to clarify this fundamental issue in prosthetics.

Address for correspondence:

Desislava Konstantinova

Department of Prosthetic Dental Medicine and Orthodontics, Faculty of Dental Medicine,

Medical University of Varna,

100 Tsar Osvoboditel Blvd, 9002 Varna, Bulgaria

e-mail:dr.konstantinova@gmail.com

Received: April 26, 2013

Accepted: July 30, 2013
The purpose of the present review is to discuss the characteristics of various types of sample foods and criteria for selection of one or another kind of test material and to display their application in certain methods of objective examination of the masticatory function.

Reviewing Bulgarian dental literature failed to identify any publications devoted to test materials. On the contrary, the number of predominantly experimental studies published by authors from Brazil, Japan and Germany permanently increases $(3,5,9,15,19)$.

Objective methods for the study of masticatory function provide specific and reliable parameters for the nature of the masticatory process. This is of importance for assessing the condition of patient's orofacial complex prior to treatment and for evaluating the outcome of prosthetic treatment as well.

Five quantitative parameters are tested such as maximum bite force, occlusive force, total number of masticatory cycles, chewing duration (in seconds) and total muscle strength (EMG area) (14). The objective methods for the examination of the masticatory function combine various techniques of precise laboratory studies by using two main types of 
food samples that meet the requirements of an objective test $(3,5,8-10,15,19,21)$.

The first type of food sample is artificial (nonedible) test material $(5,19)$, e.g. 'Optocal', 'Optocal plus', 'Optosil' (Brazilian version) (Table 1 and Fig. 1) (19).
* their structure is stable, uniform and consistent (Table 1);

* these test materials possess mechanical properties comparable to those of natural foods;

* substantial advantage is that they do not dissolve in water or saliva and can be fragmented by

Table 1. Components, trademarks, and percentages by weight of the silicon-based chewable test material of the present study

\begin{tabular}{|c|c|c|}
\hline Components & Trademark & $\begin{array}{l}\text { Percentage by } \\
\text { weight }\end{array}$ \\
\hline condensation silicon & Optosil Comfort ${ }^{\oplus}$, Heraeus Kulzer GmbH \& Co., KG, Germany & 58,3 \\
\hline $\begin{array}{l}\text { conventional tooth } \\
\text { paste }\end{array}$ & Colgate-Palmolive, Co., Osasco-SP, Brazil & 7,5 \\
\hline solid vaseline & Rioquímica, São José do Rio Preto, SP, Brazil & 11,5 \\
\hline $\begin{array}{l}\text { common powder of } \\
\text { dental plaster }\end{array}$ & $\begin{array}{l}\text { Mossoró }{ }^{\circ} \text { Empresa e Indústria Gesso Mossoró SA, Rio de Janeiro, } \\
\text { RJ, Brazil }\end{array}$ & 10,2 \\
\hline alginate powder & Jeltrate ${ }^{\circledast}$, Dentsply Indústria e Comércio Ltda., Petrópolis, RJ, Brazil & 12,5 \\
\hline mint essence & Yod Comércio de produtos naturais LTDA., Campinas, SP, Brazil & 3 drops \\
\hline
\end{tabular}

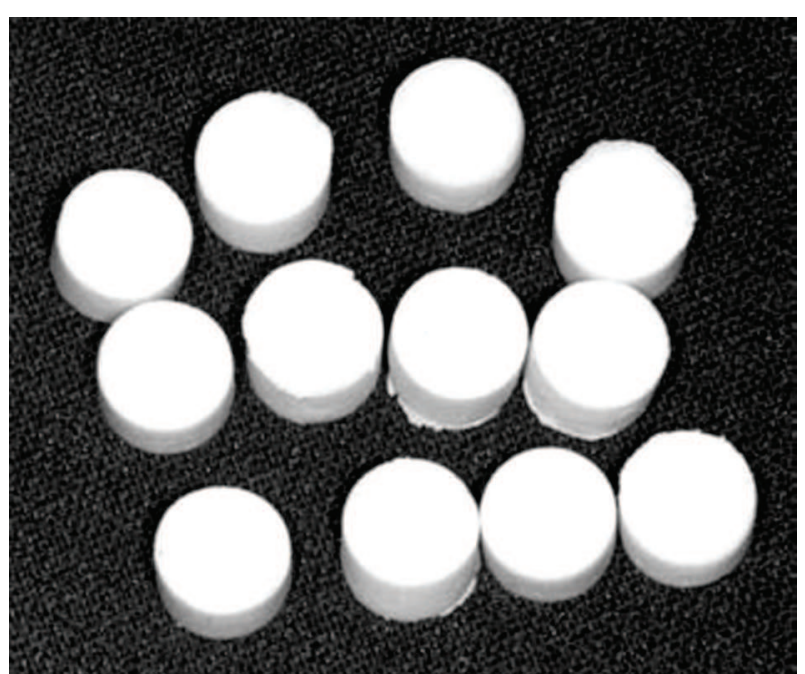

Fig. 1. Tablets of $12 \mathrm{~mm}$ in diameter and $5 \mathrm{~mm}$ in height silicon-based chewable test material (19)

Another option for sample food is Permalastic, Kep Corporation, Oringe, California, USA. It is a kind of soft rubber with dimensions $10 \times 10 \times 5 \mathrm{~mm}$ polysulphide matrix.

The detailed description found in the literature of the composition and properties of artificial test materials suggests the following advantages: chewing. Moreover, they do not break with the predefined error limit.

* they have neither taste, nor smell. They are standardized and could be stored without any quality loss.

One of the most significant disadvantages of artificial food boluses is their inability to be absorbed by the body and, therefore, not to be swallowed. This characteristics does not allow the performance of the study for the whole masticatory cycle, i.e. from biting to swallowing the food. Another disadvantage is the need of equipment and materials for their production. This results in another negative consequence, i.e. a high cost price.

Artificial sample foods are used in some objective methods associated with determining the percentage volume of masticated test material and assessed using a sieve system following a certain number of masticatory cycles. Artificial sample foods are also applied in electromyography (EMG) $(7,23)$. This method measures the bioelectrical potential of muscles ( $m$. orbicularis oris, $m$. digastricus, $m$. myohyoideus, m. geniohyoideus, etc.) (17).

Sample food texture is of importance when using this method. With a solid test material (natural 
or artificial), data are recorded for 60 seconds, whereas with liquid or paste sample food - for more than 10 and 20 seconds, respectively.

Colourimetry also uses artificial test materials containing coloured component (magenta) (18). It is quantitatively derived in $\mathrm{nm}$ Du- 640 by a UV-visible spectrophotometer (Fig. 2) (3).

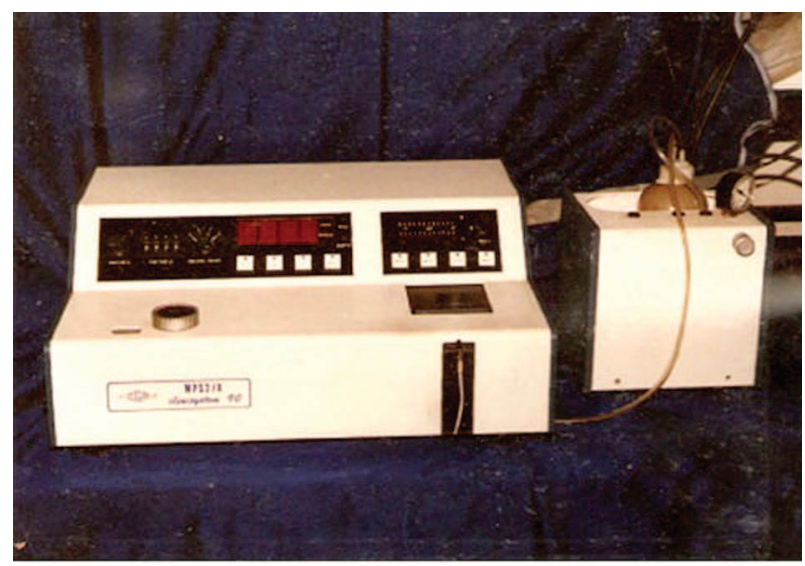

Fig. 2. Photocolourimeter after Hoeven and Kaiser (MPS2A Clinisystem)

Artificial sample foods are used by graphical methods (kinesiography). For this purpose, an electromagnetic device associated with prosthesis was developed (13).

Natural resources represent the second type of test materials $(9,15,20)$. The natural sample foods possess numerous essential advantages. They allow monitoring the whole masticatory cycle - from biting to swallowing. This enables the use of a certain method of determining the percentage volume of masticated food, namely the internationally recognized standardized system using a sieve with filter paper, whereby samples are washed and sieved through 5 sieves of 2,0,1,0,0,71, 0,5, and $0,25 \mathrm{~mm}$ of size. The particles pass through two successive sieves, indicating lower and upper margin and are assigned fractionation size. Peanuts are primarily used with this method. They are relatively uniform in size which facilitates standardization and can be used without any prior preparation. Another benefit of peanuts is that they are frequently consumed and people are accustomed to chewing them. In Bulgarian literature, these methods are known as dynamic functional methods by Hristenzen (1923), Gelman
(1932) and Rubinov (1956), or more popularly known as the chewing sample method. All of them utilize some kind of natural sample food such as hazelnuts, almonds or coconuts, etc. (8).

Another important advantage of this type of test materials is its naturally occurring dyes and colourings (necessary for colourimetry) such as raw carrots or beetroots. Natural dye can be determined spectrophotometrically at $530 \mathrm{~nm}$. This technique is preferred as it requires minimum amount of test materials, equipment and time, hence test advantages of natural foods. Equipment is not necessary for their preparation, which makes them much more accessible. They are of low price and, therefore, of a wide application range.

Conveniently, these foods produce taste and olfactory stimulus affecting the rate of chewing and swallowing. They are soluble in fluids and saliva, which in turn helps food bolus formation.

The main disadvantage of these foods is that their mechanical properties can vary as early as the first two or three seconds of the first masticatory cycle, owing to the interaction with the oral cavity. Moreover, they are not standardized in terms of shape, size, and solidity. It is noteworthy that the water content in saliva leads to texture modification. Furthermore, it can't be stored for longer periods of time due to changes in their properties. The natural foods most preferred for tests are the following: peanuts, almonds, cocoa, carrot, beetroot, jelly and decaffeinated coffee beans $(9,15,20)$.

The analysis of foreign literature sources reveals that natural test materials originate from foods exerting well-known positive effects on patient's health as well as strong preventive effects concerning socially significant diseases such as cardiovascular disease, diabetes mellitus, etc. $(9,14)$.

A detailed analysis of this group of methods emphasizes that the so-called chewing samples in all their varieties allow control of weight and size of the test material (8).

The graphic method, i. e. masticatography and kinesiography is a widely used and quite accurate objective method for examination of the masticatory efficiency $(5,8)$. The study of the masticatory process suggests the use of duplicate dentures with metal occlusal surfaces $(1,4,6)$. For the purpose of reducing 
the error factor, a prosthesis with acrylic teeth is used and, therefore, the different masticatory efficiency is due to the different materials used for acrylic denture teeth (Fig. 3) (20). Both types of test materials were applied in this experiment.

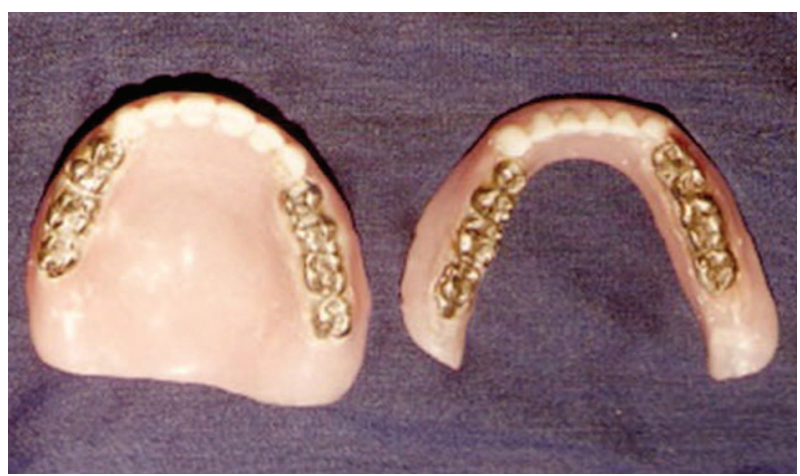

Fig. 3. Complete dentures with metal occlusal posterior teeth

An objective method of studying the masticatory process was suggested long ago (12). Using an extensometer device data were recorded from sensors in an artificial tooth of the prosthesis placed in the first molar, first premolar and second premolar. The test was carried out during mastication on shredded coconut, peanuts and raisins.

An extraoral sound transmission method was applied to measure the mechanical coupling during occlusion (11).

\section{DISCUSSION}

No universal test material for examining the masticatory function has been recognized and established yet. There does not exist any easily applicable method appropriate for clinical use. Therefore, the choice of a test material entirely depends on the purpose of the study, the state of the masticatory apparatus of the examined patient, either with a prosthesis, or edentulous, the masticatory cycle stage under examination as well as on the method used for food analysis.

Patient's capacity to grind or crush the test material by means of natural or artificial dentition is the result of complex interactions between direct and indirect forces such as number of functional units (teeth and muscles) and size of the area and dental occlusal surfaces. Therefore, the study of the masticatory function is crucial for assessing the success of prosthetic rehabilitation and patients' satisfaction concerning their masticatory abilities and associated choice of diet.

\section{CONCLUSION}

In recent years, emphasis during scientific forums and postgraduate training courses was made on numerous attempts towards 'smile design'. The importance of function and the goal for optimal performance for all denture patients was overlooked. There is a growing need for development and implementation of modern methods of diagnosis and investigation of the masticatory function providing the adequate and objective evaluation of the masticatory apparatus.

\section{REFERENCES}

1. Allen, P. F., A. S. McMillan. A review of the functional and psychosocial outcomes of edentulousness treated with complete replacement dentures.- J. Can. Dent. Assoc., 69, 2003, No 10, 662a-662e.

2. Atsuko, G., S. Kimoto, H. Koide, H. Murakami, Y. Matsumaru, K. Kimoto, et al. Investigation on how renewal of complete dentures impact on dietary and nutrient adequacy in edentulous patients.- $J$. Prosthodont. Res., 53, 2009, No 4, 180-184.

3. Awinashe, V. N., N. J. Sagda. A comparative study of the masticatory efficiency in complete dentures using acrylic and metal occlusal posterior teethphotocolorimetric analysis.- J. Indian Prosthodont. Soc., 10, 2010, No 2, 112-117.

4. Berretin-Felix, G., H. Nary Filho, C. R. Padovani, A. S. Trindade Junior, W. M. Machado. Electromyographic evaluation of mastication and swallowing in elderly individuals with mandibular fixed implant supported prostheses.- J. Appl. Oral Sci., 16, 2008, No 2, 116-121.

5. Compagnoni, M. A., R. F. de Souza, C. R. Leles. Kinesiographic study of complete denture movement related to mucosa displacement in edentulous patients.- Pesqui Odontol. Bras., 17, 2003, No 4, 356-361.

6. Davies, S., R. Gray, J. F. McCord. Good occlusal practice in removable prosthodontics.- Br. Dent. J., 191, 2001, No 9, 492-502.

7. de Las Casas, E. B., A. F. de Almeida, C. A. Cimini Junior, T. Gomes Pde, T. P. Cornacchia, J. M. Saffar. Determination of tangential and normal com- 
ponents of oral forces.- J. Appl. Oral Sci., 15, 2007, No 1, 70-76.

8. Filchev, A., R. Ralev. Introduction to clinical prosthetic dentistry. Sofia, Medical University Sofia, 2010, 158-160 (in Bulgarian).

9. Fraser, G. E., J. Sabaté, W. L. Beeson, T. M. Strahan. A possible protective effect of nut consumption on risk of coronary heart disease. The Adventist Health Study.- Arch. Intern. Med., 152, 1992, No 7, 1416-1424.

10. Fueki K., E. Yoshida, K. Okano, Y. Igarashi. Association between occlusal curvature and masticatory movements with different test foods in human young adults with permanent dentitions.Arch. Oral Biol., 58, 2013, No 6, 674-680.

11. Gibbs, C. H., P. E. Mahan, H. C. Lundeen, K. Brehnan, E. K. Walsh, W. B. Holbrook. Occlusal forces during chewing and swallowing as measured by sound transmission.- J. Prosthet. Dent., 46, 1981, No 4, 443-449.

12. Howell, A. H., F. Brudevold. Vertical forces used during chewing of food.- J. Dent. Res., 29, 1950, No 2, 133-136.

13. Maeda, Y., M. Okada, A. Makishi, T. Nokubi, Y. Okuno, T. Aoki. Using mandibular kinesiograph for measuring complete denture movements - a preliminary report.- J. Osaka Univ. Dent. Sch., 24, 1984, 123-129.

14. McKiernan, F., R. D. Mattes. Effects of peanut processing on masticatory performance during variable appetitive states.- J. Nutr. Metab., 2010; 2010, pii: 487301. doi: 10.1155/2010/487301.

15. Moon, S., K. Jae, S. Beom, S. Heung. Masticatory function following implants replacing a second molar.- J. Periodont. Implant Sci., 41, 2011, No 2, 79-85.
16. Muller, K., J. Morais, J. Feine. Nutritional and anthropometric analysis of edentulous patients wearing implant overdentures or conventional dentures.- Braz. Dent. J., 19, 2008, No 2, 145-150.

17. Neill, D. J. The relationship between masticatory performance and diet.- Proc. Roy. Soc. Med., 66, 1973, No 6, 598-599.

18. Picinato-Pirola M. N., W. Mestriner Jr., O. Freitas, F. V. Mello-Filho, L. V. Trawitzki. Masticatory efficiency in class II and class III dentofacial deformities.- Int. J. Oral Maxillofac. Surg., 41, 2012, No 7, 830-834.

19. Pocztaruk Rde, L., L. C. Frasca, E. G. Rivaldo, L. Fernandes Ede, M. B. Gavião. Protocol for production of a chewable material for masticatory function tests (Optocal - Brazilian version).- Braz. Oral Res., 22, 2008, No 4, 305-310.

20. Takeshita, S., M. Kanazawa, S. Minakuchi. Stress analysis of mandibular two-implant overdenture with different attachment systems.- Dent. Mater. J., 30, 2011, No 6, 928-934.

21. Witter, D. J., A. Woda, E. M. Bronkhorst, N. H. Creugers. Clinical interpretation of a masticatory normative indicator analysis of masticatory function in subjects with different occlusal and prosthodontic status.- J. Dent., 41, 2013, No 5, 443-448.

22. Wöstmann, B., K. Michel, B. Brinkert, A. Melchheier-Weskott, P. Rehmann, M. Balkenhol. Influence of denture improvement on the nutritional status and quality of life of geriatric patients.- $J$. Dent., 36, 2008, No 10, 816-821.

23. Yurkstas, A., R. S. Manly. Value of different test foods in estimating masticatory ability.- J. Appl. Physiol., 3, 1950, No 1, 45-53. 